VENTURA I OLLER Montserrat, Identité, cosmologie et chamanisme des Tsachila de l'Équateur

Istvan Praet

\title{
OpenEdition
}

Journals

Édition électronique

URL : https://journals.openedition.org/jsa/12006

DOI : $10.4000 /$ jsa. 12006

ISSN : 1957-7842

Éditeur

Société des américanistes

Édition imprimée

Date de publication : 20 décembre 2011

Pagination : 439-441

ISSN : 0037-9174

\section{Référence électronique}

Istvan Praet, " VENTURA I oller Montserrat, Identité, cosmologie et chamanisme des Tsachila de l'Équateur », Journal de la Société des américanistes [En ligne], 97-2 | 2011, mis en ligne le 22 décembre 2011, consulté le 07 septembre 2022. URL : http://journals.openedition.org/jsa/12006 ; DOI : https://doi.org/ $10.4000 / j s a .12006$

Ce document a été généré automatiquement le 4 septembre 2022

Tous droits réservés 


\title{
VENTURA I OLLER Montserrat, Identité, cosmologie et chamanisme des Tsachila de l'Équateur
}

\author{
Istvan Praet
}

\section{RÉFÉRENCE}

VENTURA I OLLER Montserrat, Identité, cosmologie et chamanisme des Tsachila de l'Équateur, préface de Jean-Pierre Chaumeil, L'Harmattan, coll. « Recherches Amériques latines », Paris, 2009, 286 p.

Dans cet excellent livre, Montserrat Ventura i Oller entame une recherche sur une société à la croisée de deux mondes qui fascinent les ethnologues américanistes depuis longtemps : les civilisations andines et les sociétés « sauvages » des Basses Terres. Bien que, dans quelques travaux récents, les relations entre les sociétés des Hautes Terres et celles des Basses Terres de l'Amérique du Sud aient été examinées, il n'existe à ce jour que peu d'analyses entièrement consacrées à l'étude de la confluence de ces deux genres de société que tout semble distinguer. C'est l'une des innovations majeures de cet ouvrage sur les Tsachila du piémont occidental des Andes équatoriennes.

2 Habitants d'une région subtropicale proche de la ville de Santo Domingo, une zone intermédiaire entre la côte pacifique et les Andes, les Tsachila étaient autrefois connus sous le nom de Colorado, parce qu'ils avaient l'habitude de se peindre en rouge avec le roucou (Bixa orellana). En fait, beaucoup d'hommes tsachila continuent de se teindre les cheveux en rouge; de nos jours, c'est un de leurs principaux atouts touristiques, qui a, par exemple, contribué à leur faire tenir le rôle d'Indiens supposément guarani dans le film de Roland Joffe, «Mission» (palme d'or à Cannes, 1986). Dans la première partie du livre, "Chroniques à la croisée », l'auteur décrit l'histoire coloniale de la région. Elle explique l'importance de la route entre Quito, la capitale, et Santo Domingo, construite entre 1920 et 1940. De façon détaillée, elle explore le rôle de l'État et les effets de la ley de 
tierras baldías y colonización de 1936, élément essentiel pour comprendre les conflits actuels au sujet des ressources et des limites des terres tsachila, que les colons ont souvent considérées comme «non cultivées ».

Bien que l'éventail des contacts se soit énormément élargi après la construction de la route vers la côte pacifique, Ventura défend la thèse que les Tsachila ont déployé un réseau de rapports avec l'extérieur tout au long de leur histoire. De manière convaincante, elle montre que les documents coloniaux attestent les échanges entre les habitants de l'actuelle région des Tsachila et les différentes ethnies des forêts côtières, des Hautes Terres andines et, même, de l'Amazonie. Ces échanges ne concernent d'ailleurs pas seulement des biens matériels comme le coton, l'or ou le piment rouge (souvent troqués contre du sel ou des marchandises d'origine hispanique), mais aussi des savoirs chamaniques et des plantes médicinales. Loin d'être une tribu isolée, les Tsachila ont toujours été en contact étroit avec des représentants coloniaux, notamment les cobradores de tributos. Mais, à la différence des révoltes violentes qui ont été décrites ailleurs en Amérique du Sud, la stratégie principale des Tsachila semble avoir été la fuite.

Dans la deuxième partie du livre, «La personne », Ventura insiste sur le fait que, hormis la dispersion ou l'indifférence, on ne connaît pas de rejet explicite des fonctionnaires coloniaux, ni des curés missionnaires. Son argument mérite d'être cité en détail : « Le pacifisme tsachila, qui guide toutes les pratiques culturelles, mène à la résolution des conflits par la voie de la retraite, plus que la confrontation ouverte. Ainsi, l'indignation ou le désaccord avec les Métis se manifeste souvent par le silence et la disparition » (p.137). Elle tient à nous avertir que cela ne doit pas être confondu avec de la passivité ou de la lâcheté: "... la timidité associée à cette conduite d'évasion ne signale pas la soumission dans son sens strict. Bien au contraire, c'est la fierté qui guide ces résolutions. Se taire face aux Espagnols fut aussi une pratique qui mena les Tsachila dans leur actuelle région de refuge. Et malgré la spoliation territoriale, aucune révolte indienne n'a été rapportée par l'histoire écrite. [...] La fuite, l'évitement du conflit, ne peuvent pourtant pas être expliqués de façon satisfaisante par l'assaut colonial. Ces conduites font partie, à mon avis, d'un ethos culturel poussé à l'extrême lors des moments de crise du contact interethnique » (pp.137-138). Cette observation perspicace est en parfaite conformité avec mes propres conclusions sur les Chachi - groupe indigène voisin - qui ont, eux aussi, développé l'évitement comme réponse systématique (Praet 2009 ; voir aussi Lorcy 2010). Ventura souligne à juste titre que la relation entre Tsachila et Chachi a été étroite à travers l'histoire et l'est encore aujourd'hui. Dans ce cas également, la relation économique se double d'une relation d'apprentissage chamanique. Il s'avère que le chamanisme est une des voies principales de rapports interethniques.

5 La troisième et dernière partie du livre, « Les chemins du cosmos ", est consacrée au rôle des pone, les chamanes qui sont des maîtres guérisseurs extrêmement réputés à travers l'Équateur, même parmi les Métis et les Quichua de la sierra. De manière presque poétique, Ventura décrit comment ces chamanes protègent les gens d'une pléiade de créatures pernicieuses comme luban oko, une sorte de vampire rouge, ou sonpura, un spectre du monde souterrain ayant l'apparence d'un tatou. Elle esquisse ainsi les chemins entre les deux mondes de la cosmologie tsachila : celui des vivants et celui des morts. Ce qui émerge dans l'avant-dernier chapitre (« Un fil de vie»), c'est que les Tsachila ont une notion de la vie riche et complexe. Toutefois, je ne suis pas sûr que Ventura soit allée jusqu'au bout sur ce point précis. Dans sa compréhension de l'animisme tsachila, la vie s'étend à l'ensemble des choses qui constituent ce qu'on appelle aujourd'hui la biosphère 
et même au-delà (incluant la lune, le soleil, les astres). En revanche, je me demande si leur notion de la vie n'est pas beaucoup plus restreinte, à l'instar de leur notion de l'humanité que Ventura a analysée admirablement (pp.50-56). Sur ce point, il convient de souligner que tsachila signifie, littéralement, « les véritables gens ». Autrement dit, les non-Tsachila seraient (ou, au moins, auraient été) exclus de l'humanité. À proprement parler, les étrangers (Quichua, Blancs...) n'étaient pas des "autres humains », mais des "non-humains ". Je soupçonne même qu'ils n'étaient pas conçus comme des "autres êtres vivants ", mais plutôt comme des " êtres non vivants ». La généralisation est peutêtre excessive, mais toujours est-il que, chez les Chachi, les étrangers (ainsi que les animaux sauvages) appartiennent au monde des morts, plutôt qu'à celui des vivants (Praet s. d.). Espérons que les futures enquêtes de Ventura éclairent la question. documentation d'une richesse exceptionnelle et un travail de terrain prolongé. Le ton est vif, incisif et parfois critique, mais jamais prétentieux ou pédant. Il s'agit donc, globalement, d'une réalisation ethnographique tout à fait remarquable. Ventura établit une nouvelle norme pour les recherches sur la côte pacifique de l'Équateur. Elle met la barre très haut.

\section{BIBLIOGRAPHIE}

LORCY Armelle

2010 «Cuisiner les sensibilités ». Alimentation, affects et société (Noirs et Indiens Chachi du littoral équatorien), thèse d'ethnologie, Université Paris-Ouest Nanterre-La Défense, Nanterre.

\section{PRAET Istvan}

2009 "Shamanism and ritual in South America. An inquiry into Amerindian shape-shifting », Journal of the Royal Anthropological Society, 15 (4), pp. 737-754.

s. d. "Humanity and life among Chachi and other so-called indigenous people », in Tim Ingold et Gísli Pálsson (éds), Biosocial becomings, Cambridge University Press, Cambridge (MA) [à paraître].

\section{AUTEURS}

\section{ISTVAN PRAET}

Roehampton University, Londres/Rachel Carson Centre, Munich 